\title{
Recurrent osteosarcoma with a single pulmonary metastasis: a multi-institutional review
}

\author{
N C Daw ${ }^{*, 1}$, A J Chou ${ }^{2}$, N Jaffe ${ }^{1}$, B N Rao ${ }^{3,4}$, C A Billups ${ }^{5}$, C Rodriguez-Galindo ${ }^{6,7,8}$, P A Meyers ${ }^{2}$ and W W Huh ${ }^{1}$ \\ ${ }^{1}$ Division of Paediatrics, The University of Texas MD Anderson Cancer Center, Houston, TX 77030, USA; ${ }^{2}$ Department of \\ Paediatrics, Memorial Sloan-Kettering Cancer Center, New York, NY 10065, USA; ${ }^{3}$ Department of Surgery, St Jude Children's \\ Research Hospital, Memphis, TN 38105, USA; ${ }^{4}$ Department of Surgery, College of Medicine, University of Tennessee Health \\ Science Center, Memphis, TN 38163, USA; ${ }^{5}$ Department of Biostatistics, St Jude Children's Research Hospital and the University of \\ Tennessee, Memphis, TN 38105, USA; ${ }^{6}$ Department of Oncology, St Jude Children's Research Hospital, Memphis, TN 38105, USA \\ and ${ }^{7}$ Department of Paediatrics, College of Medicine, University of Tennessee Health Science Center, Memphis, TN 38105, USA
}

Background: Late relapse and solitary lesion are positive prognostic factors in recurrent osteosarcoma.

Methods: We reviewed the records of 39 patients treated at three major centres for recurrent osteosarcoma with a single pulmonary metastasis more than 1 year after diagnosis. We analysed their outcomes with respect to clinical factors and treatment with chemotherapy.

Results: Median age at diagnosis was 14.6 years. Relapse occurred at a median of 2.5 years (range, 1.2-8.2 years) after initial diagnosis. At relapse, all patients were treated by metastasectomy; 12 (31\%) patients also received chemotherapy. There was no difference in time to recurrence or nodule size between the patients who received or did not receive chemotherapy at relapse. Sixteen patients had no subsequent recurrence, 13 of whom survive without evidence of disease. The 5-year and 10-year estimates of post-relapse event-free survival (PREFS) were $33.0 \pm 7.5 \%$ and $33.0 \pm 9.6 \%$, respectively, and of post-relapse survival (PRS) $56.8 \pm 8.6 \%$ and $53.0 \pm 11.0 \%$, respectively. There was a trend for nodules $<1.5 \mathrm{~cm}$ to correlate positively with PREFS $(P=0.070)$ but not PRS $(P=0.49)$. Chemotherapy at first relapse was not associated with PREFS or PRS.

Conclusion: Approximately half of the patients with recurrent osteosarcoma presenting as a single pulmonary metastasis more than 1 year after diagnosis were long-term survivors. Metastasectomy was the primary treatment; chemotherapy did not add benefit.

Despite aggressive surgery and intensive chemotherapy, osteosarcoma recurs in $\sim 30-40 \%$ of patients initially diagnosed with nonmetastatic disease (Meyer et al, 2001; Meyers et al, 1998; Meyers et al, 2005; Kempf-Bielack et al, 2005; Bacci et al, 2005b,c; Whelan et al, 2012). The outcome of patients with recurrent osteosarcoma is generally poor, with long-term survival rates of $\sim 20 \%$ (Ferrari et al, 2003; Hawkins and Arndt, 2003; Kempf-Bielack et al, 2005; Bacci et al, 2005a). The most common site of relapse is the lung, and $50-75 \%$ of patients with recurrent osteosarcoma initially have lung involvement only (Chi et al, 2004; Crompton et al, 2006;
Gelderblom et al, 2011). Favourable prognostic indicators in recurrent disease include a longer disease-free interval from initial diagnosis to relapse, good histologic response to neoadjuvant chemotherapy in the primary tumour, a solitary lesion, and the ability to achieve a second complete surgical remission (Ferrari et al, 2003; Kempf-Bielack et al, 2005; Gelderblom et al, 2011; Leary et al, 2013). For patients with pulmonary relapse, fewer nodules, unilateral involvement, complete surgical resection, and absence of pleural disruption are favourable prognostic factors (Briccoli et al, 2005; Kempf-Bielack et al, 2005; Harting et al, 2006).

*Correspondence: Dr NC Daw; E-mail: ndaw@mdanderson.org

${ }^{8}$ Current address: Department of Paediatric Oncology, Dana-Farber/Children's Hospital Cancer Center, Boston, MA 02215, USA

Received 3 September 2014; accepted 27 October 2014; published online 25 November 2014

(C) 2015 Cancer Research UK. All rights reserved 0007 - 0920/15 
Although adjuvant chemotherapy is necessary for the treatment of newly diagnosed osteosarcoma, the role of chemotherapy in the treatment of recurrent disease is uncertain, especially for patients with solitary recurrent pulmonary metastasis. A study demonstrated that a disease-free interval $>12$ months before developing osteosarcoma lung metastasis and complete surgical metastasectomy correlated with increased overall survival (OS; Harting et al, 2006). Therefore, patients with recurrent osteosarcoma presenting with a single pulmonary nodule more than 1 year after diagnosis may represent a group of patients with favourable outcome for whom chemotherapy does not add benefit. The goals of this study were to characterise the clinical features and outcome of these patients and to evaluate the role of chemotherapy in this setting.

\section{PATIENTS AND METHODS}

After obtaining approval from the Institutional Review Board of each participating institution, a retrospective medical record review was conducted at three institutions: Memorial Sloan-Kettering Cancer Center, St Jude Children's Research Hospital (SJCRH), and the Children's Cancer Hospital of the University of Texas MD Anderson Cancer Center. Patients were considered eligible for this study if they were younger than 21 years of age at the time of initial diagnosis of non-metastatic high-grade osteosarcoma and had recurrence with only a single pulmonary metastasis more than 1 year after diagnosis that was confirmed by histology at the time of thoracotomy. Data collected included patient demographics, anatomic site of primary tumour, chemotherapy agents used during initial therapy, grade of histologic response to neoadjuvant therapy using the criteria described by Huvos (1991), time from diagnosis to recurrence, pulmonary nodule size, treatment at the time of recurrence, patient outcome, duration of follow-up, and cause of death. The date of relapse was defined as the time of thoracotomy for tumour removal.

Post-relapse survival (PRS) was defined as the time interval from the date of relapse to the date of death from any cause or the date of last contact for survivors. Post-relapse event-free survival (PREFS) was defined as the time interval from the date of relapse to the date of subsequent relapse or death from any cause or to the date of last contact for patients without subsequent events. PRS and PREFS were estimated using the method of Kaplan and Meier. Differences in distributions of PRS and PREFS among categorical variables were investigated using the exact log-rank test. Cox proportional hazards model was used to examine the association between continuous variables and outcome. Exact Wilcoxon ranksum tests were used to examine the association between chemotherapy use at relapse with time to recurrence and nodule size.

\section{RESULTS}

Patient characteristics. A total of 39 patients satisfied the study inclusion criteria; their characteristics are summarised in Table 1. The median age at initial diagnosis was 14.6 years (range, 6.0-20.0 years). All patients were confirmed to have localised disease at the time of diagnosis. The femur was the most common primary site $(N=22 ; 56 \%)$.

Treatment. The patients were diagnosed between October 1977 and November 2002, and received different chemotherapy during this 25-year period (Table 1). For primary therapy, all patients had surgery and all but one received chemotherapy. The median number of active chemotherapeutic agents received was three (range, 0-4). For 31 patients with available data, the median time from date of diagnosis to date of definitive surgery was 2.3 months (range, 0-60 months), and 8 of these patients had surgery within
Table 1. Patient characteristics and initial treatment

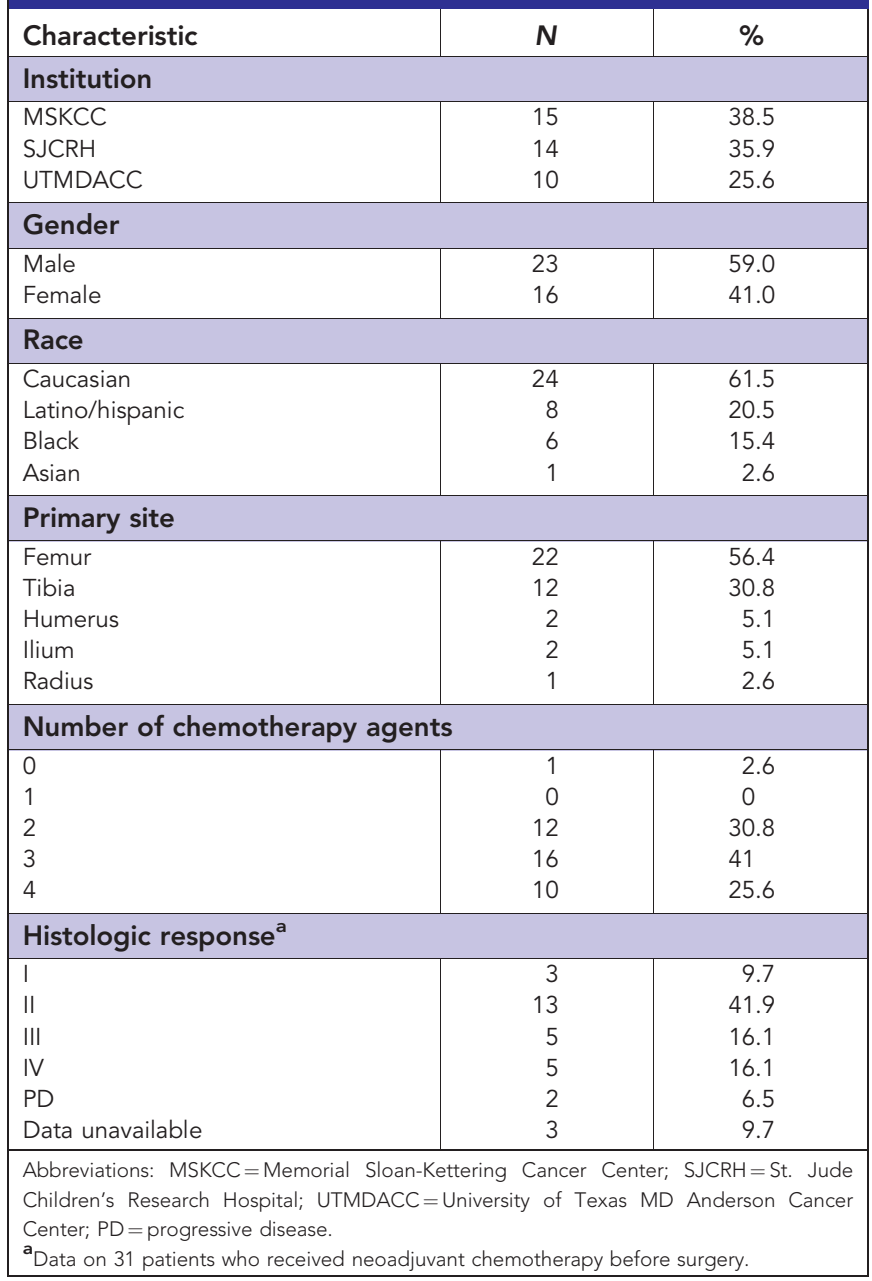

10 days of diagnosis. For the 31 patients who did not have upfront surgery as part of their primary treatment, histologic responses were grade I $(N=3)$, grade II $(N=13)$, grade III $(N=5)$, and grade IV $(N=5)$; progressive disease occurred before surgery in 2 patients and no data was available for 3 patients.

Recurrence and treatment at recurrence. All patients had a thoracotomy; the median time from diagnosis to thoracotomy date (recurrence date) was 2.5 years (range, 1.2-8.2 years; (Figure 1). The nodules occurred in the left $(N=22)$ or right $(N=17)$ lung. In 36 patients with available data, the median largest dimension of the pulmonary nodule was $1.5 \mathrm{~cm}$ (range, $0.5-18 \mathrm{~cm}$ ). Twelve $(31 \%)$ patients received chemotherapy at relapse.

Patient outcome. Twenty-three of 39 patients (59\%) experienced a second recurrence, and 13 of these patients (57\%) had a third recurrence or disease progression. Table 2 shows the timing of recurrence for patients who experienced disease recurrence. Twenty-one of 39 patients (54\%) were alive at the time of analyses. The median follow-up duration from initial diagnosis was 12 years (range, 1.8-22.1 years). The median follow-up duration from the date of thoracotomy for survivors was 7.7 years (range, 0.6-20.9 years). Eighteen patients had no evidence of disease and three were alive with disease. The remaining 18 patients died of disease $(N=14)$ or other causes: acute myeloid leukaemia $(N=1)$, brain aneurysm $(N=1)$, sepsis $(N=1)$, and unknown cause $(N=1)$. The 5 -year and 10-year estimates of PREFS were $33.0 \pm 7.5 \%$ and $33.0 \pm 9.6 \%$, respectively, and of PRS $56.8 \pm 8.6 \%$ and $53.0 \pm 11.0 \%$, respectively (Figure 2 ). 
Prognostic factors. Table 3 summarises the results of investigating various factors as predictors of PRS and PREFS. None of the factors investigated were found to be significantly predictive of PRS. When investigated as a continuous variable, there was no evidence that time from diagnosis to relapse was significantly predictive of PRS $(P=0.123$; hazard ratio, 0.75 (95\% confidence interval, $0.53-1.08)$ ). When investigated as a categorical variable $(\geqslant 1$ year to $<2$ years $v s \geqslant 2$ years to $<3$ years $v s \geqslant 3$ years to $<4$ years $v s \geqslant 4$ years after diagnosis), a trend was observed towards a positive predictive value for longer duration between diagnosis and relapse for PREFS $(P=0.062)$, but not for PRS $(P=0.31)$. When analysed as a continuous variable, nodule size was not significantly associated with PRS ( $P=0.80$; hazard ratio, 0.98). When studied as a categorical variable, a trend was observed between size $<1.5 \mathrm{~cm}$ and improved PREFS $(P=0.070)$, but not PRS $(P=0.49)$. Chemotherapy at first relapse did not affect PREFS $(P=0.84)$ or PRS $(P=0.54)$. Further analysis was performed to investigate whether there was any selection bias for patients who received chemotherapy at relapse with respect to nodule size and time to recurrence. There was no statistically significant difference for time to recurrence and nodule size between patients who received chemotherapy and those who did not $(P>0.11)$.

\section{DISCUSSION}

In this study, we analysed the treatment and outcome of 39 patients treated at three major cancer centres for osteosarcoma that recurred as a single pulmonary metastasis more than 1 year after diagnosis. All patients had thoracotomy and about one third received chemotherapy. The 10-year PREFS and PRS were $33.0 \pm 9.6 \%$ and $53.0 \pm 11.0 \%$, respectively. These survival estimates compare favourably with those of patients with first relapse of osteosarcoma in general (10-year PREFS, 12\% and 10-year PRS, 17-18\%; Kempf-Bielack et al, 2005, Leary et al, 2013). Our study

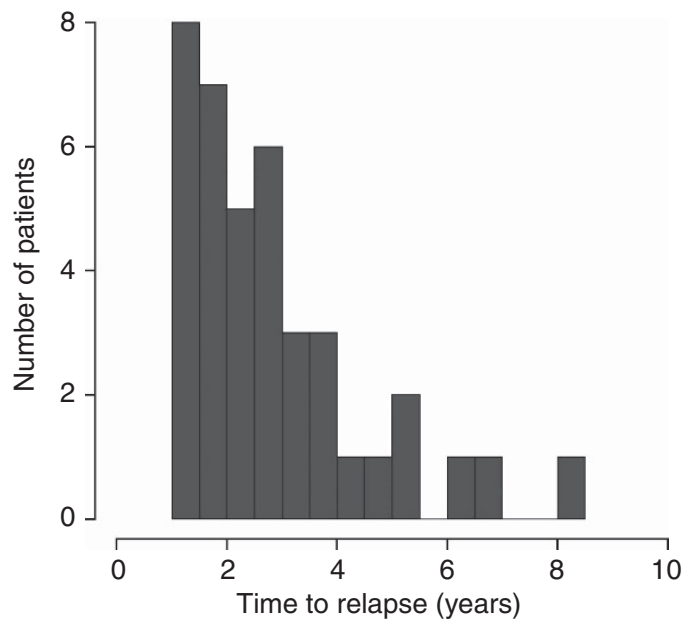

Figure 1. Distribution of interval time from diagnosis to recurrence (thoracotomy) for 39 patients with recurrent osteosarcoma presenting as a single pulmonary metastasis. confirms that patients with recurrent osteosarcoma presenting as a single pulmonary nodule more than 1 year after diagnosis represent a favourable group of patients who can be cured by surgery alone. The use of chemotherapy at first relapse did not improve outcome.

In a retrospective review of 576 patients with relapsed osteosarcoma by the Cooperative Osteosarcoma Study Group (COSS), $80 \%$ of patients had lung metastasis and $65 \%$ of patients had lung metastasis as the only site of recurrence (Kempf-Bielack et al, 2005). Another review by the European Osteosarcoma Intergroup noted lung-only recurrence in $54 \%$ of the patients (Gelderblom et al, 2011). Although the long-term prognosis for patients with recurrent osteosarcoma remains poor, some progress has been made in identifying risk factors that can be used for patient stratification and tailoring salvage therapy. Hawkins and Arndt (2003) reported that for patients with initial pulmonary recurrence, the presence of solitary pulmonary nodules was associated with improved disease-free survival and OS. The COSS study demonstrated that bilateral pulmonary metastases and pleural disruption were poor prognostic factors (Kempf-Bielack et al, 2005). Leary et al (2013) reported that for patients with pulmonary relapse, unilateral disease and the presence of $\leqslant 3$ nodules were favourable clinical factors.

Surgical resection of pulmonary metastases, whenever feasible, has been long considered standard treatment (Carter et al, 1991, van Geel et al, 1996, Harting et al, 2006). Disease-free interval following metastasectomy appears to be important for survival in recurrent osteosarcoma in the lung; one study noted a $3 \%$ decrease in mortality associated with each additional month of disease-free interval (Briccoli et al, 2005). Thoracotomy with wedge resection has been the preferred surgical approach for removal of lung nodules; a median sternotomy does not allow full exploration of the posterior pulmonary segments for nodules that may not have been radiologically evident. All patients in our study underwent thoracotomy for removal of the lung nodule. However, a recent study suggested that a minimally invasive approach to nodule removal with image-guided localisation, if needed, should be considered in patients with single pulmonary nodule because ipsilateral metastases are not likely to be found (Fernandez-Pineda et al, 2012).

The use of chemotherapy for patients with isolated pulmonary recurrence of osteosarcoma has been widely debated. One significant consideration for using a surgical-only treatment approach is to avoid the potential acute and late toxicities of chemotherapy. In the Hawkins and Arndt (2003) study, survival was superior among patients with pulmonary recurrence treated with surgery alone compared with patients treated with chemotherapy and surgery $(47 \%$ vs $13 \%, P=0.005)$. For patients with isolated pulmonary recurrence who achieved a second complete remission of disease, a trend towards improved survival rates for patients treated with surgery alone approached statistical significance $(45 \%$ vs $13 \%, P=0.08)$. However, the improved survival for patients treated with surgery alone likely reflects a biased use of chemotherapy in patients with incompletely resected disease. Ferrari et al (2003) reported that PRS was positively influenced by the use of second-line chemotherapy in patients who did not have complete surgery, but not in patients who had

\begin{tabular}{|l|c|c|c|}
\hline & $\mathbf{N}$ & Median & Range \\
\hline Time from diagnosis to RL1 & 39 & 2.5 years & 1.2 years-8.2 years \\
\hline Time from thoracotomy to RL2 & 23 & 10.8 months & 1.8 months-3.8 years \\
\hline Time from RL2 to RLPD3 & 13 & 5.2 months & 1.6 months-5.9 years \\
\hline Abbreviations: RL1 = first relapse; RL2= second relapse; RLPD3=third relapse or disease progression. \\
\hline
\end{tabular}


complete surgery. In their subgroup analysis of patients with isolated pulmonary recurrence who had complete surgical remission, PRS was better in patients with two or fewer nodules and a relapse-free

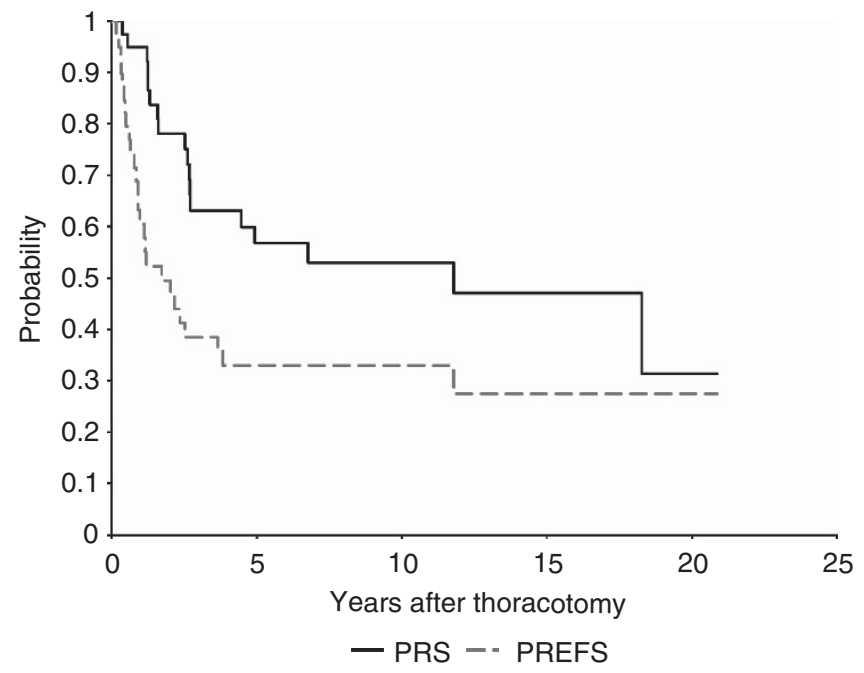

Figure 2. Post-relapse survival and event-free survival for 39 patients with recurrent osteosarcoma presenting as a single pulmonary metastasis. interval longer than 24 months. In the COSS study of the largest cohort of patients with recurrent osteosarcoma, the use of secondline chemotherapy, particularly chemotherapy with more than one agent, was associated with better OS for the entire cohort and for patients without second complete remission, but not for patients with second complete remission (Kempf-Bielack et al, 2005). In patients who achieved a second complete remission, second-line chemotherapy positively correlated with event-free survival but not OS. In the SJCRH experience with 110 patients with relapsed osteosarcoma, the use of chemotherapy at first relapse was associated with improved PREFS only in patients without second complete remission, and was not associated with PRS (Leary et al, 2013). Another study based on single institutional experience did not demonstrate a survival advantage for those patients who received chemotherapy compared with patients who only underwent surgical resection for first recurrence (Crompton et al, 2006). Analysis of our cohort did not reveal survival advantage with the use of chemotherapy. Thus, the lack of evidence for benefit from salvage chemotherapy and the relatively favourable outcome of patients with relapsed osteosarcoma presenting as a single pulmonary nodule support the use of surgery only for treating these patients.

Our study is limited by its retrospective nature and the small cohort size that may have affected our ability to further delineate whether some of the clinical prognostic factors would have reached statistical significance. Because our study included only patients who had a relapse more than 1 year after diagnosis and the median time to

Table 3. Exploratory analyses of potential prognostic factors

\begin{tabular}{|c|c|c|c|c|c|c|c|}
\hline & & \multicolumn{3}{|c|}{ PRS \pm s.e. (\%) } & \multicolumn{3}{|c|}{ PREFS \pm s.e. (\%) } \\
\hline Factors & $\mathbf{N}$ & Year 5 & Year 10 & $P$ & Year 5 & Year 10 & $P$ \\
\hline \multicolumn{8}{|l|}{ Age at diagnosis } \\
\hline$<16$ years & 27 & $62.1 \pm 10.2$ & $56.4 \pm 12.4$ & \multirow[t]{2}{*}{0.86} & $32.8 \pm 9.0$ & $32.8 \pm 11.0$ & \multirow[t]{2}{*}{0.78} \\
\hline$\geq 16$ years & 12 & $46.3 \pm 13.9$ & $46.3 \pm 19.6$ & & $33.3 \pm 12.2$ & $33.3 \pm 15.7$ & \\
\hline \multicolumn{8}{|l|}{ Gender } \\
\hline Female & 16 & $57.7 \pm 12.5$ & $57.7 \pm 14.2$ & \multirow[t]{2}{*}{0.93} & $40.4 \pm 11.8$ & $40.4 \pm 13.9$ & \multirow[t]{2}{*}{0.49} \\
\hline Male & 23 & $55.9 \pm 11.2$ & $49.7 \pm 15.8$ & & $27.8 \pm 8.9$ & $27.8 \pm 11.8$ & \\
\hline \multicolumn{8}{|l|}{ Primary tumour site } \\
\hline Femur & 22 & $56.1 \pm 10.7$ & $49.9 \pm 14.4$ & \multirow[t]{2}{*}{0.83} & $33.8 \pm 9.7$ & $33.8 \pm 12.3$ & \multirow[t]{2}{*}{0.61} \\
\hline Other bones & 17 & $58.2 \pm 13.3$ & $58.2 \pm 15.4$ & & $31.9 \pm 10.7$ & $31.9 \pm 13.2$ & \\
\hline \multicolumn{8}{|l|}{ No. of active agents } \\
\hline$<3$ active agents & 13 & $59.8 \pm 13.4$ & $49.9 \pm 17.7$ & \multirow[t]{2}{*}{0.78} & $30.8 \pm 11.4$ & $30.8 \pm 14.8$ & \multirow[t]{2}{*}{0.95} \\
\hline$\geq 3$ active agents & 26 & $55.5 \pm 10.7$ & $55.5 \pm 13.1$ & & $34.3 \pm 9.3$ & $34.3 \pm 11.3$ & \\
\hline \multicolumn{8}{|l|}{ Histologic response } \\
\hline Grade I or II or PD & 18 & $46.4 \pm 12.8$ & $46.4 \pm 15.2$ & \multirow[t]{2}{*}{0.73} & $20.0 \pm 8.9$ & $20.0 \pm 10.3$ & \multirow[t]{2}{*}{0.80} \\
\hline Grade III or IV & 10 & $52.5 \pm 16.2$ & $52.5 \pm 25.6$ & & $30.0 \pm 12.5$ & $30.0 \pm 17.7$ & \\
\hline \multicolumn{8}{|l|}{ Time to thoracotomy } \\
\hline$\geq 1$ year to $<2$ years & 15 & $43.1 \pm 13.3$ & $43.1 \pm 14.5$ & \multirow[t]{4}{*}{0.31} & $22.2 \pm 9.8$ & $22.2 \pm 9.8$ & \multirow[t]{4}{*}{0.062} \\
\hline$\geq 2$ years to $<3$ years & 11 & $62.5 \pm 15.6$ & $46.9 \pm 17.1$ & & $18.2 \pm 9.5$ & $18.2 \pm 11.6$ & \\
\hline$\geq 3$ years to $<4$ years & 6 & $50.0 \pm 17.7$ & $50.0 \pm 25.0$ & & $33.3 \pm 15.7$ & $33.3 \pm 19.2$ & \\
\hline$\geq 4$ years & 7 & $83.3 \pm 13.9$ & $83.3 \pm 19.6$ & & $85.7 \pm 13.2$ & $85.7 \pm 18.7$ & \\
\hline \multicolumn{8}{|l|}{ Time to thoracotomy } \\
\hline$<18$ months & 8 & $42.9 \pm 16.2$ & $42.9 \pm 16.2$ & \multirow[t]{2}{*}{0.97} & $30.0 \pm 14.5$ & $30.0 \pm 14.5$ & \multirow[t]{2}{*}{0.93} \\
\hline$\geq 18$ months & 31 & $60.3 \pm 9.5$ & $55.2 \pm 13.1$ & & $33.7 \pm 8.3$ & $33.7 \pm 11.2$ & \\
\hline \multicolumn{8}{|c|}{ Site of pulmonary nodule } \\
\hline Left lung & 22 & $54.7 \pm 11.1$ & $54.7 \pm 16.5$ & \multirow[t]{2}{*}{0.95} & $34.1 \pm 9.8$ & $34.1 \pm 13.8$ & \multirow[t]{2}{*}{0.66} \\
\hline Right lung & 17 & $58.9 \pm 12.6$ & $51.6 \pm 13.6$ & & $31.5 \pm 10.6$ & $31.5 \pm 11.7$ & \\
\hline \multicolumn{8}{|c|}{ Size of Pulmonary Nodule } \\
\hline$<1.5 \mathrm{~cm}$ & 17 & $59.2 \pm 12.6$ & $50.8 \pm 14.5$ & \multirow[t]{2}{*}{0.49} & $44.1 \pm 11.7$ & $44.1 \pm 13.5$ & 0.070 \\
\hline$\geq 1.5 \mathrm{~cm}$ & 19 & $57.7 \pm 11.9$ & $57.7 \pm 16.8$ & & $28.5 \pm 9.8$ & $28.5 \pm 13.9$ & \\
\hline Chemotherapy at $f$ & & & & & & & \\
\hline Yes & 12 & $50.9 \pm 14.6$ & $50.9 \pm 17.8$ & 0.54 & $37.5 \pm 13.3$ & $37.5 \pm 17.1$ & 0.84 \\
\hline No chemotherapy & 27 & $59.0 \pm 10.1$ & $54.1 \pm 13.0$ & & $31.2 \pm 8.6$ & $31.2 \pm 10.6$ & \\
\hline
\end{tabular}


relapse was 2.5 years, we could not further ascertain whether patients who present with a solitary pulmonary nodule recurrence may in fact represent a subset of relapsed patients with longer duration of diseasefree interval, a known good prognostic factor. In the COSS study, a correlation was noted between solitary site of relapse and longer interval to relapse (Kempf-Bielack et al, 2005).

In conclusion, our multi-institutional study demonstrated that approximately half of the patients with recurrent osteosarcoma as a solitary pulmonary nodule more than 1 year after diagnosis were long-term survivors. Whether this improved outcome compared with that of patients with recurrent osteosarcoma in general could be related to low disease burden or favourable tumour biology remains to be determined. Our analyses did not reveal factors that help identify patients who are likely to survive or die, although there was a trend for improved outcome for those with smaller nodules or later relapse. It is hoped that clinical genomics or the ability to detect circulating sarcoma cells will help discern which patients will require further therapy in addition to surgery (Chen et al, 2014, Satelli et al, 2014). While the use of salvage chemotherapy did not confer a survival advantage in our study, perhaps novel therapies that are likely to be effective in the setting of minimal residual disease such as immunotherapy, will prove to be beneficial.

\section{ACKNOWLEDGEMENTS}

This work was supported in part by Cancer Centre Support (CORE) grant CA21765 and grant CA23099 from the National Cancer Institute and by the American Lebanese Syrian Associated Charities (ALSAC).

\section{CONFLICT OF INTEREST}

The authors declare no conflict of interest.

\section{REFERENCES}

Bacci G, Briccoli A, Longhi A, Ferrari S, Mercuri M, Faggioli F, Versari M, Picci P (2005a) Treatment and outcome of recurrent osteosarcoma: experience at Rizzoli in 235 patients initially treated with neoadjuvant chemotherapy. Acta Oncol 44: 748-755.

Bacci G, Longhi A, Fagioli F, Briccoli A, Versari M, Picci P (2005b) Adjuvant and neoadjuvant chemotherapy for osteosarcoma of the extremities: 27 year experience at Rizzoli Institute, Italy. Eur J Cancer 41: $2836-2845$.

Bacci G, Mercuri M, Longhi A, Ferrari S, Bertoni F, Versari M, Picci P (2005c) Grade of chemotherapy-induced necrosis as a predictor of local and systemic control in 881 patients with non-metastatic osteosarcoma of the extremities treated with neoadjuvant chemotherapy in a single institution. Eur J Cancer 41: 2079-2085.

Briccoli A, Rocca M, Salone M, Bacci G, Ferrari S, Balladelli A, Mercuri M (2005) Resection of recurrent pulmonary metastases in patients with osteosarcoma. Cancer 104: 1721-1725.

Carter S, Grimer R, Sneath R, Matthews HR (1991) Results of thoracotomy in osteogenic sarcoma with pulmonary metastases. Thorax 46: 727-731.

Chen X, Bahrami A, Pappo A, Easton J, Dalton J, Hedlund E, Ellison D, Shurtleff S, Wu G, Wei L, Parker M, Rusch M, Nagahawatte P, Wu J, Mao S, Boggs K, Mulder H, Yergeau D, Lu C, Ding L, Edmonson M, Qu C, Wang J, Li Y, Navid F, Daw N, Mardis E, Wilson R, Downing JR, Zhang J, Dyer M, St. Jude Children's Research Hospital Washnington University Pediatric Cancer Genome (2014) Recurrent somatic structural variations contribute to tumorigenesis in pediatric osteosarcoma. Cell Rep 7: 104-112.

Chi S, Conklin L, Qin J, Meyers P, Huvos A, Healey J, Gorlick R (2004) The patterns of relapse in osteosarcoma: the Memorial Sloan-Kettering experience. Pediatr Blood Cancer 42: 46-51.
Crompton B, Goldsby R, Weinberg V, Feren R, O’Donnell R, Ablin A (2006) Survival after recurrence of osteosarcoma: a 20-year experience at a single institution. Pediatr Blood Cancer 47: 255-259.

Fernandez-Pineda I, Daw N, McCarville B, Emanus L, Rao B, Davidoff A, Shochat S (2012) Patients with osteosarcoma with a single pulmonary nodule on computed tomography: a single-institution experience. J Pediatr Surg 47: 1250-1254.

Ferrari S, Briccoli A, Mercuri M, Bertoni F, Picci P, Tienghi A, Del Prever A, Fagioli F, Comandone A, Bacci G (2003) Postrelapse survival in osteosarcoma of the extremities: prognostic factors for long-term survival. J Clin Oncol 21: 710-715.

Gelderbolm H, Jinks R, Sydes M, Bramwell V, Van Glabbeke M, Grimer R, Hogendoorn P, McTiernan A, Lewis I, Nooij M, Taminiau A, Whelan J (2011) Survival after recurrent osteosarcoma: data from 3 European Osteosarcoma Intergroup (EOI) randomized controlled trials. Eur J Cancer 47: 895-902.

Harting M, Blakely M, Jaffe N, Cox Jr C, Hayes-Jordan A, Benjamin R, Raymond A, Andrassy R, Lally K (2006) Long-term survival after aggressive resection of pulmonary metastases among children and adolescents with osteosarcoma. J Pediatr Surg 41: 194-199.

Hawkins D, Arndt C (2003) Pattern of disease recurrence and prognostic factors in patients with osteosarcoma treated with contemporary chemotherapy. Cancer 98: 2447-2456.

Huvos A (1991) Pathologic assessment of preoperative (neoadjuvant) chemotherapy. In Bone Tumors: Diagnosis, treatment, and prognosis. 2nd edn. W.B. Saunders: Philadelphia, PA, USA.

Kempf-Bielack B, Bielack S, Jurgens H, Branscheid D, Berdel W, Exner G, Gobel U, Helmke K, Jundt G, Kabisch H, Kevric M, Klingebiel T, Kotz R, Maas R, Schwarz R, Semik M, Treuner J, Zoubek A, Winkler K (2005) Osteosarcoma relapse after combined modality therapy: an analysis of unselected patients in the Cooperative Osteosarcoma Study Group (COSS). J Clin Oncol 23: 559-568.

Leary S, Wozniak A, Billups C, Wu J, McPherson V, Neel M, Rao B, Daw N (2013) Survival of pediatric patients after relapsed osteosarcoma: the St. Jude Children's Research Hospital experience. Cancer 119: 2645-2653.

Meyer W, Pratt C, PoQuette C, Rao B, Parham D, Marina N, Pappo A, Mahmoud H, Jenkins J, Harper J, Neel M, Fletcher B (2001) Carboplatin/ifosfamide window therapy for osteosarcoma: results of the St Jude Children's Research Hospital OS-91 trial. J Clin Oncol 19: 171-182.

Meyers P, Gorlick R, Heller G, Casper E, Lane J, Huvos A, Healey J (1998) Intensification of preoperative chemotherapy for osteogenic sarcoma: results of the Memorial Sloan-Kettering (T12) protocol. J Clin Oncol 16: 2452-2458.

Meyers P, Schwartz C, Krailo M, Kleinerman E, Betcher D, Bernstein M, Conrad E, Ferguson W, Gebhardt M, Goorin A, Harris M, Healey J, Huvos A, Link M, Montebello J, Nadel H, Nieder M, Sato J, Siegal G, Weiner M, Wells R, Wold L, Womer R, Grier H (2005) Osteosarcoma: a randomized, prospective trial of the addition of ifosfamide and/or muramyl tripeptide to cisplatin, doxorubicin, and high-dose methotrexate. J Clin Oncol 23: 2004-2011.

Satelli A, Mitra A, Cutrera J, Devarie M, Xia X, Ingram D, Dibra D, Somaiah N, Torres K, Ravi V, Ludwig J, Kleinerman E, Li S (2014) Universal marker and detection tool for human sarcoma circulating tumor cells. Cancer Res 74: 1645-1650.

van Geel A, Pastorino U, Jauch K, Judson I, Van Coevorden F, Buesa J, Nielsen O, Boudinet A, Tursz T, Schmitz P (1996) Surgical treatment of lung metastases: The European Organization for Research and Treatment of Cancer-Soft Tissue and Bone Sarcoma Group study of 255 patients. Cancer 77: 675-682.

Whelan J, Jinks R, McTiernan A, Sydes M, Hook J, Trani L, Uscinska B, Bramwell V, Lewis I, Nooij M, Van Glabbeke M, Grimer R, Hogendoorn P, Taminiau A, Gelderblom H (2012) Survival from high-grade localised extremity osteosarcoma: combined results and prognostic factors from three European Osteosarcoma Intergroup randomised controlled trials. Ann Oncol 23: 1607-1616.

This work is published under the standard license to publish agreement. After 12 months the work will become freely available and the license terms will switch to a Creative Commons AttributionNonCommercial-Share Alike 3.0 Unported License. 